\title{
Differentiation of chronic mass-forming pancreatitis from pancreatic ductal adenocarcinoma using contrast-enhanced computed tomography
}

This article was published in the following Dove Press journal: Cancer Management and Research

\author{
Shuai Ren (D) \\ Xiao Chen' \\ Wenjing Cui' \\ Rong Chen ${ }^{2}$ \\ Kai Guo' \\ Huifeng Zhang' \\ Shuai Chen' \\ Zhongqiu Wang (1D' \\ 'Department of Radiology, The Affiliated \\ Hospital of Nanjing University of Chinese \\ Medicine, Nanjing, Jiangsu Province \\ 210029, People's Republic of China; \\ ${ }^{2}$ Department of Diagnostic Radiology and \\ Nuclear Medicine, University of Maryland \\ School of Medicine, Baltimore, MD \\ 2I20I, USA
}

Correspondence: Zhongqiu Wang Department of Radiology, The Affiliated Hospital of Nanjing University of Chinese Medicine, No. 155 Hanzhong Road, Nanjing, Jiangsu Province 210029, People's Republic of China

Tel +86258086 I278

Email zhqwang00I@I26.com
Purpose: Both chronic mass-forming pancreatitis (CMFP) and pancreatic ductal adenocarcinoma (PDAC) are focal pancreatic lesions and share very similar clinical symptoms and imaging performance. There is great clinical value in preoperative differentiation of those two lesions. The purpose of this study was to investigate the value of computed tomography (CT) features in discriminating CMFP from PDAC.

Patients and methods: Forty-seven patients with pathologically confirmed PDAC and 21 patients with CMFP were included in this study. Demographic and CT features, including tumor location, size, margin, pancreatic or bile duct dilatation, vascular invasion, cystic necrosis, pancreatic atrophy, calcification, and tumor contrast enhancement, were retrospectively analyzed and compared. Multivariate logistic regression analyses were adopted to identify relevant CT imaging features to discriminate CMFP from PDAC.

Results: There were significant differences between CMFP and PDAC with respect to main pancreatic duct dilatation, vascular invasion, cystic necrosis, pancreatic atrophy, calcification, and tumor contrast enhancement. Delayed contrast enhancement ( $>70.5$ Hounsfield units) showed high sensitivity and specificity of $84.2 \%$ and $84.7 \%$. The areas under the curve (AUCs) of the predicting models based on qualitative and quantitative variables were 0.770 (95\% CI: $0.660-0.880)$ and 0.943 (95\% CI: 0.888-0.999), respectively. When all significant variables were used in combination to build a predicting model, the AUC was $0.969(95 \%$ CI: $0.930-1.000$ ) with $84.2 \%$ sensitivity and $94.7 \%$ specificity.

Conclusion: Main pancreatic duct dilatation, vascular invasion, cystic necrosis, pancreatic atrophy, calcification, tumor size, and tumor contrast enhancement were shown to be useful CT imaging features in discriminating CMFP from PDAC.

Keywords: pancreas, adenocarcinoma, pancreatitis, computed tomography

\section{Introduction}

Pancreatic ductal adenocarcinoma (PDAC), the most common primary malignancy of pancreas, is a major cause of cancer-associated mortality, with a dismal overall prognosis that has remained virtually unchanged for many decades. ${ }^{1}$ Therefore, early diagnosis is very important for PDAC patients due to its aggressive course and high mortality. Moreover, it can help to increase the survival rate by providing a chance for surgical treatment and adjuvant intervention. ${ }^{2,3}$

Chronic mass-forming pancreatitis (CMFP) is a special type of chronic pancreatitis associated with autoimmune reaction, long-term alcohol drinking, and a history of biliary disease, which accounts for $10-30 \%$ of chronic pancreatitis. ${ }^{4}$ 
CMFP must be differentiated accurately from PDAC as both may present with clinically recurrent abdominal pain, weight loss, pancreatic insufficiency, and a pancreatic mass on imaging. However, CMFP and PDAC require entirely different treatment strategies and management and have different prognoses. Sometimes, tissue sampling has its limitations in differentiating those two entities. Given the small amount of material aspirated in these biopsies, obtaining an accurate representation of heterogeneous and large neoplasms can be challenging. ${ }^{5}$ Therefore, noninvasive imaging modalities including computed tomography (CT), magnetic resonance imaging (MRI), magnetic resonance cholangiopancreatography, and 18F-FDG positron emission tomography (PET)/CT play crucial roles in accurate differential diagnosis, personalized treatment, and evaluation of therapeutic effects in both entities. ${ }^{4,6-11}$

$\mathrm{CT}$ is the most appropriate initial imaging modality for evaluation of patients with suspected chronic pancreatitis, depicting most changes in pancreatic morphology. ${ }^{12}$ Several studies have demonstrated that perfusion CT or dual-energy CT is a useful technology that can be helpful in differentiating CMFP from PDAC. ${ }^{13-15}$ However, there remain some hospitals that do not carry out perfusion CT or dual-energy CT examinations in their daily clinical practice. Few studies demonstrated the value of contrastenhanced CT (CE-CT) in morphological evaluation and contrast enhancement properties for differentiating CMFP from PDAC.

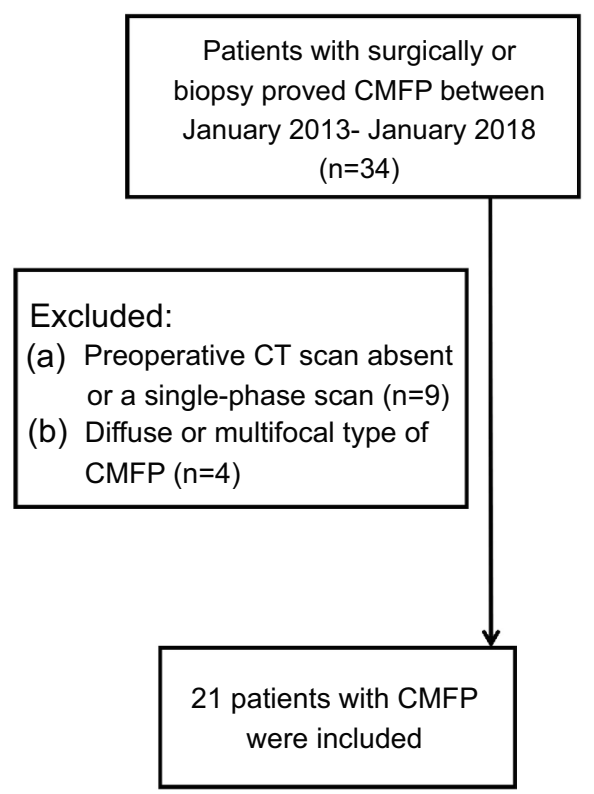

The purpose of this study was to investigate the potential of CE-CT for diagnostic differentiation between CMFP and PDAC and provide strategies for clinicians with non-invasive imaging.

\section{Materials and methods}

\section{Patient selection}

This study was approved by the institutional review board of the Affiliated Hospital of Nanjing University of Chinese Medicine and patient informed consent was waived due to its retrospective nature. All analyses of confidential data were carried out in accordance with the Declaration of Helsinki principles. Thirty-four patients with pathologically proved CMFP through fine-needle aspiration cytology or postoperative specimen between January 2013 and January 2018 were enrolled in our study. Exclusion criteria were as follows: a) preoperative CT scan absent or a single-phase scan $(n=9)$ or (b) diffuse or multifocal type of CMFP $(n=4)$. Finally, 21 patients (12 men and 9 women) were included in our study (Figure 1). The mean age was $60.14 \pm 7.82$ years (age range, 49-75).

Similarly, 73 patients with pathologically proved PDAC through fine-needle aspiration cytology or postoperative specimen between January 2016 and January 2018 were enrolled in our study. Exclusion criteria were as follows: a) preoperative CT scan absent or a singlephase scan $(\mathrm{n}=17)$; or b) tumor presented as dominantly

Patients with surgically or biopsy proved PDAC between January 2016- January 2018 $(n=73)$

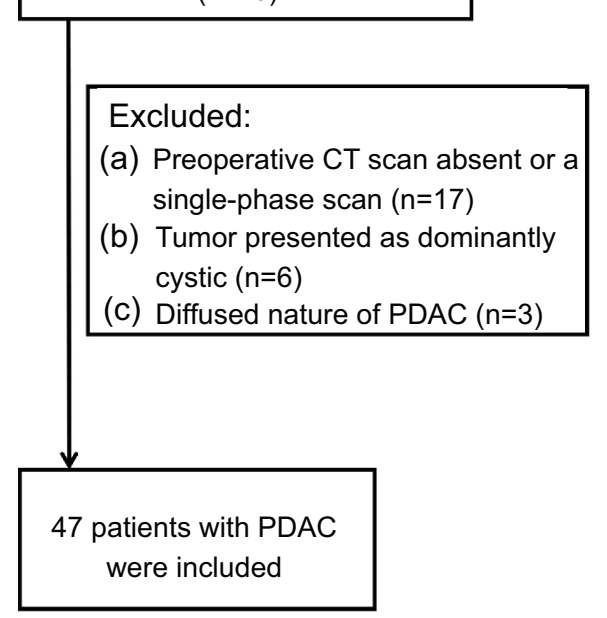

Figure I Flow diagram showing inclusion and exclusion criteria of the study population.

Abbreviations: CT, computed tomography; CMFP, chronic mass-forming pancreatitis; PDAC, pancreatic ductal adenocarcinoma. 
cystic $(n=6)$; or $c)$ diffused nature of PDAC $(n=3)$. Finally, 47 patients (26 men and 21 women) were included in our study (Figure 1). The mean age was $64.53 \pm 9.06$ years (age range, 39-78 years).

\section{CT imaging}

All pathologically diagnosed patients underwent multidetector spiral CT (MSCT) examinations. Unenhanced and threephase contrast material enhanced CT examinations were performed by using the Discovery HD750 (GE Healthcare, Milwaukee, WI, USA), Philips Brilliance 64 (Philips Healthcare, DA Best, the Netherlands) and optima 670 (GE Healthcare, Tokyo, Japan). MSCT scanning was performed through the elbow vein of patients with the help of a power injector (Ulrich Medical, Berlin, Germany). A total of $80-100 \mathrm{~mL}$ of nonionic contrast media (Ultravist 300, Bayer Schering Pharma AG, Berlin, Germany ) with $1.2 \mathrm{~mL} / \mathrm{kg}$ of body weight was administrated at a rate of 3.0 $\mathrm{mL} / \mathrm{s}$ followed by $40 \mathrm{~mL}$ saline solution. The CT scanning parameters were as follows: tube voltage of $120 \mathrm{kVp}$; tube current of 200-400 mAs; a helical pitch of 1.375 ; slice thickness of $3.0 \mathrm{~mm}$; slice interval of $3.0 \mathrm{~mm}$; and a reconstruction interval of $1.25 \mathrm{~mm}$. Nineteen cases of CMFP and 39 cases of PDAC patients underwent a 4phase CT examination (unenhanced, arterial, portal venous, and delayed phase). The other two cases of CMFP and eight cases of PDAC patients underwent a 3-phase CT examination (unenhanced, arterial, and portal venous phase). The enhanced images were obtained at the arterial phase (30 s), the portal venous phase $(60 \mathrm{~s})$, and the delayed phase (120 s).

\section{CT imaging analysis Qualitative analysis}

Two experienced abdominal radiologists (with $>8$ years of experience in abdominal CT reading) who were blinded to the detailed histopathological information of any patient reviewed CT images independently. Any inconformity during image analysis was resolved by discussion or referral to a third radiologist with 13 years of experience in abdominal CT reading. Qualitative analysis included the following features: a) tumor location (head and neck vs body and tail), b) tumor margin (well-defined vs ill-defined), c) main pancreatic duct dilatation (uniformity of dilatation vs beaded dilatation), d) bile duct dilatation, e) vascular invasion, f) cystic necrosis, g) pancreatic atrophy, and h) calcification. A smooth and clearly visible margin indicated a well-defined margin, while spiculation or infiltration on $>90^{\circ}$ of tumor perimeter indicated a poorly defined margin. ${ }^{3}$ Both the extrahepatic bile duct $(>8 \mathrm{~mm})$ and the intrahepatic bile duct $(>2 \mathrm{~mm})$ indicated bile duct dilatation. ${ }^{16}$ Vascular invasion was defined as the fat disappeared between the mass and adjacent vessels, the mass wrapped around more than $180^{\circ}$ of the circumference of the adjacent vessels, and blood vessels were observed with stenosis. ${ }^{17}$ Cystic necrosis was defined as a lack of contrast enhancement within the tumor at the arterial, portal venous, and delayed phase. ${ }^{18}$ Pancreatic atrophy was defined as atrophy of parenchyma distal to the focal lesion or disproportional atrophy which was associated with age. ${ }^{19}$ Calcification was identified at the unenhanced images.

\section{Quantitative analysis}

The third radiologist with 13 years of experience in abdominal $\mathrm{CT}$ reading measured the size $(\mathrm{cm})$ and $\mathrm{CT}$ attenuation [Hounsfield units (HU)] of the tumors and the adjacent parenchyma. The regions of interest (ROIs) were manually drawn on the solid part of tumor showing the most remarkable enhancement and the downstream parenchyma. During the measurements of CT attenuation, intratumoral calcification and cystic necrosis were avoided and the ROIs at the other phases were drawn on the same equivalent sections. CT attenuation was measured three times by placing the ROIs of each lesion as large as possible. We then calculated the mean $\mathrm{CT}$ attenuation by calculating an average for the ROI measurements. The tumor-to-pancreas enhancement ratio was calculated by dividing the $\mathrm{CT}$ attenuation (HU) of pancreatic lesion by that of the pancreatic parenchyma at each phase of CE-CT.

\section{Statistical analysis}

All statistical analyses were performed with commercially available software (SPSS, Version 20.0 IBM Corp., Chicago, IL, USA IBM SPSS Statistics for Windows). The prevalence of each CT finding between CMFP and PDAC was compared by using Chi-square or Fisher's exact tests for categorical variables and the Student $t$ or Mann-Whitney U test for quantitative variables. Kappa analysis was used to evaluate the concordance of the qualitative variables between the two readers. Multivariate logistic regression analyses were adopted to determine differential independent $\mathrm{CT}$ features, and the receiver operating characteristic (ROC) analysis was performed to ascertain diagnostic ability, sensitivity, and specificity for the regression models in differentiating CMFP 
from PDAC. A $P$-value $<0.05$ was considered to indicate a significant difference.

\section{Results}

\section{Patient and tumor characteristics}

Characteristics of the $21 \mathrm{CMFP}$ and 47 PDAC patients are summarized in Table 1. Twenty-one patients with pathologically confirmed CMFP through surgery (17 cases) or fine-needle aspiration cytology (4 cases) and 47 patients with pathologically confirmed PDAC through surgery (43 cases) or fine-needle aspiration cytology (4 cases) were included and compared in our study. There were no significant differences between the two groups with respect to age, gender, or most of the clinical symptoms. However, abdominal pain was more common in PDAC compared with CMFP patients (51.1\% vs $33.3 \%$ ), but no significant difference was observed. Notably, a history of pancreatitis was more common in CMFP than that of PDAC $(81.0 \%$ vs $19.1 \%, P<0.001)$.

\section{Comparison of CT features between CMFP and PDAC}

\section{Qualitative analysis}

Qualitative CT findings between CMFP and PDAC patients are summarized in Table 2. No significant differences were found in tumor location, margin, or bile duct dilatation between CMFP and PDAC patients. PDAC showed pancreatic duct dilatation more frequently than did CMFP (61.7\% [29 of 47] vs 33.3\% [7 of 21],
$P=0.031)$. Notably, uniformity of dilatation was more common in PDAC than that of CMFP (57.4\% [27 of 47] vs $4.8 \%$ [1 of 21], $P<0.001)$ and beaded dilatation was more common in CMFP than that of PDAC $(28.6 \%$ [6 of 21] vs $4.3 \%$ [2 of 47], $P=0.014$ ). Moreover, vascular invasion, cystic necrosis, and pancreatic atrophy were more frequent in PDAC than those of CMFP (all $P<0.05)$. Vascular invasion was found in $53.2 \%(25 / 47)$ of PDAC, cystic necrosis was found in 59.6\% (28/47) of PDAC, and pancreatic atrophy was found in $55.3(26 / 47)$ of PDAC, while only $23.8 \%$ (5/21), 28.6\% (6/21), and $19.0 \%$ (4/21), respectively, of CMFP exhibited such. PDAC showed calcification less frequently than did CMFP (10.6\% [5 of 47] vs 33.3\% [7 of 21], $P=0.023$ ). The kappa values for those qualitative variables were $>0.80$. Figure 2 shows a representative case of CMFP, which demonstrated focal enlargement of the pancreatic head (Figure 2A) without pancreatic atrophy (Figure 2D), and calcifications were clearly seen in the pancreatic head tumor. Figure 3 shows a representative case of PDAC, which demonstrated a pancreatic head tumor (Figure 3A) with pancreatic atrophy and upstream duct dilatation (Figure 3D). As was shown in Figure 4, CT attenuation of CMFP was higher than that of PDAC at the arterial, portal venous, and delayed phase. Figure 4A-D shows a gradual enhancement pattern, Figure 4E-H shows a hypovascular enhancement pattern. Besides, Figure 4E also demonstrated a pancreatic body tumor with pancreatic tail atrophy.

Table I Demographic characteristics of patients with chronic mass-forming pancreatitis (CMFP) vs pancreatic ductal adenocarcinoma (PDAC)

\begin{tabular}{|l|l|l|l|}
\hline Variables & CMFP (n=2I) & PDAC (n=47) & P-value \\
\hline Age (years) & $60.14 \pm 7.82$ & $64.53 \pm 9.06$ & $0.059^{\mathrm{a}}$ \\
\hline $\begin{array}{l}\text { Sex } \\
\text { Male }\end{array}$ & $12(57.1 \%)$ & $26(53.3 \%)$ & $0.889^{\mathrm{b}}$ \\
Female & $9(42.9 \%)$ & $21(44.7 \%)$ & \\
\hline $\begin{array}{l}\text { Clinical symptom } \\
\text { Abdominal pain }\end{array}$ & $7(33.3 \%)$ & $24(51.1 \%)$ & \\
Abdominal bloating or diarrhea & $5(23.8 \%)$ & $17(36.2 \%)$ & $0.175^{\mathrm{b}}$ \\
Yellow urine or icterus & $4(19.0 \%)$ & $11(23.4 \%)$ & $0.314^{\mathrm{b}}$ \\
History of pancreatitis & $17(81.0 \%)$ & $9(19.1 \%)$ & $0.689^{\mathrm{b}}$ \\
Asymptomatic & $3(14.3 \%)$ & $7(14.9 \%)$ & $<0.001^{\mathrm{b}}$ \\
\hline Surgery & $17(81.0 \%)$ & $43(91.5 \%)$ & $0.948^{\mathrm{b}}$ \\
\hline Biopsy & $4(19.0 \%)$ & $4(8.5 \%)$ & $0.402^{\mathrm{b}}$ \\
\end{tabular}

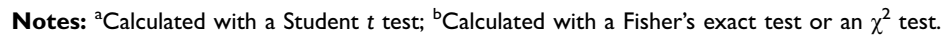


Table 2 Comparisons of CT findings between chronic mass-forming pancreatitis (CMFP) and pancreatic ductal adenocarcinoma (PDAC)

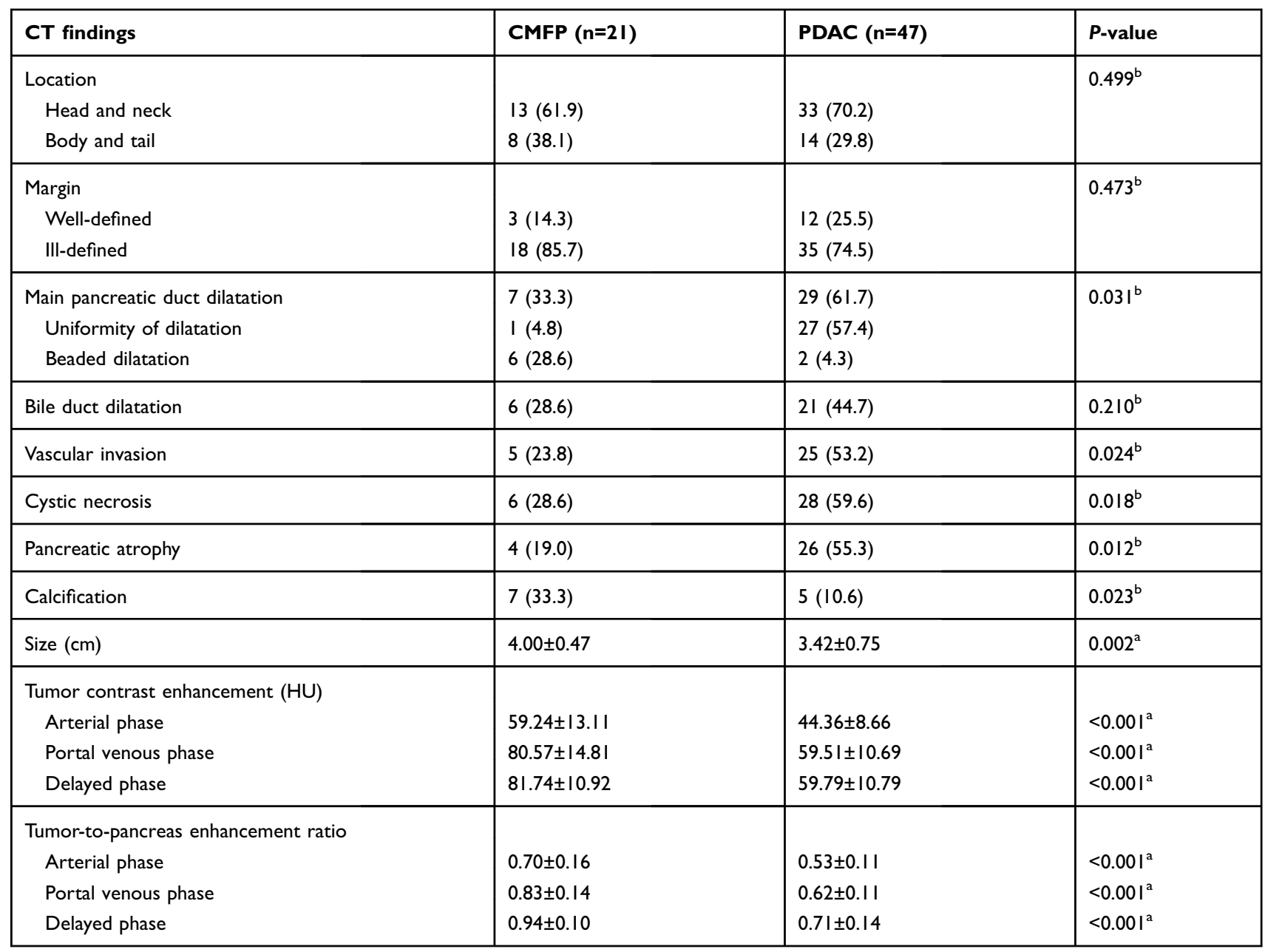

Notes: ${ }^{a}$ Calculated with a Student $t$ or Mann-Whitney $U$ test; ${ }^{b}$ Calculated with a Fisher's exact test or an $\chi^{2}$ test.

\section{Quantitative analysis}

Quantitative CT findings between CMFP and PDAC are summarized in Table 2. The size of CMFP was significantly larger than that of PDAC $(4.00 \pm 0.47 \mathrm{~cm}$ vs $3.42 \pm 0.75 \mathrm{~cm}$, $P=0.002)$.The attenuation of CMFP at the arterial phase (59.24 $\pm 13.11 \mathrm{HU}$ vs $44.36 \pm 8.66 \mathrm{HU})$, portal venous phase (80.57 $\pm 14.81 \mathrm{HU}$ vs $59.51 \pm 10.69 \mathrm{HU})$, and delayed phase (81.74 $\pm 10.92 \mathrm{HU}$ vs $59.79 \pm 10.79 \mathrm{HU})$ was significantly higher than that of PDAC (all $P<0.001$ ) (Figure 5). The tumor-to-pancreas enhancement ratios of CMFP at the arterial phase $(0.70 \pm 0.16$ vs $0.53 \pm 0.11)$, portal venous phase $(0.83 \pm 0.14$ vs $0.62 \pm 0.11$ ), and delayed phase $(0.94 \pm 0.10$ vs $0.71 \pm 0.14)$ were significantly higher than those of PDAC (all $P<0.001$ ) (Figure 6).

\section{Diagnostic performance of CT findings in discriminating CMFP from PDAC}

Multivariate logistic regression analyses for discriminating CMFP from PDAC are shown in Table 3. For qualitative variables, main duct dilatation $[(\mathrm{OR}=1.741 ; 95 \% \mathrm{CI}$ : $1.085-2.792 ; P=0.03)$, pancreatic atrophy $(\mathrm{OR}=1.812 ; 95 \%$ CI: $1.239-2.649 ; P=0.024)$, and calcification $(\mathrm{OR}=2.746$; 95\% CI: $0.543-1.025 ; P=0.023)$ were independent predictors of differentiation between CMFP and PDAC. For quantitative variables, delayed contrast enhancement $(\mathrm{OR}=0.833$; 95\% CI: $0.757-0.917 ; P<0.001)$ and tumor size (OR $=0.151 ; 95 \%$ CI: $0.028-0.815 ; P=0.028)$ were independent predictors of differentiation between CMFP and PDAC. We built two predicting models based on qualitative (Model 1) and quantitative variables (Model 2) for the differentiation of CMFP from PDAC, respectively. ROC curves and AUCs of predicting models in the differentiation of those two tumors are demonstrated in Figure 7. For quantitative variables, the cutoff values were $70.5 \mathrm{HU}$ with $84.2 \%$ sensitivity and $84.7 \%$ specificity and $3.285 \mathrm{~cm}$ with $100 \%$ sensitivity and $50.9 \%$ specificity. The AUCs of Model 1 and Model 2 were 0.770 (95\% CI: $0.660-0.880)$ and 0.943 (95\% CI: $0.888-0.999)$, 


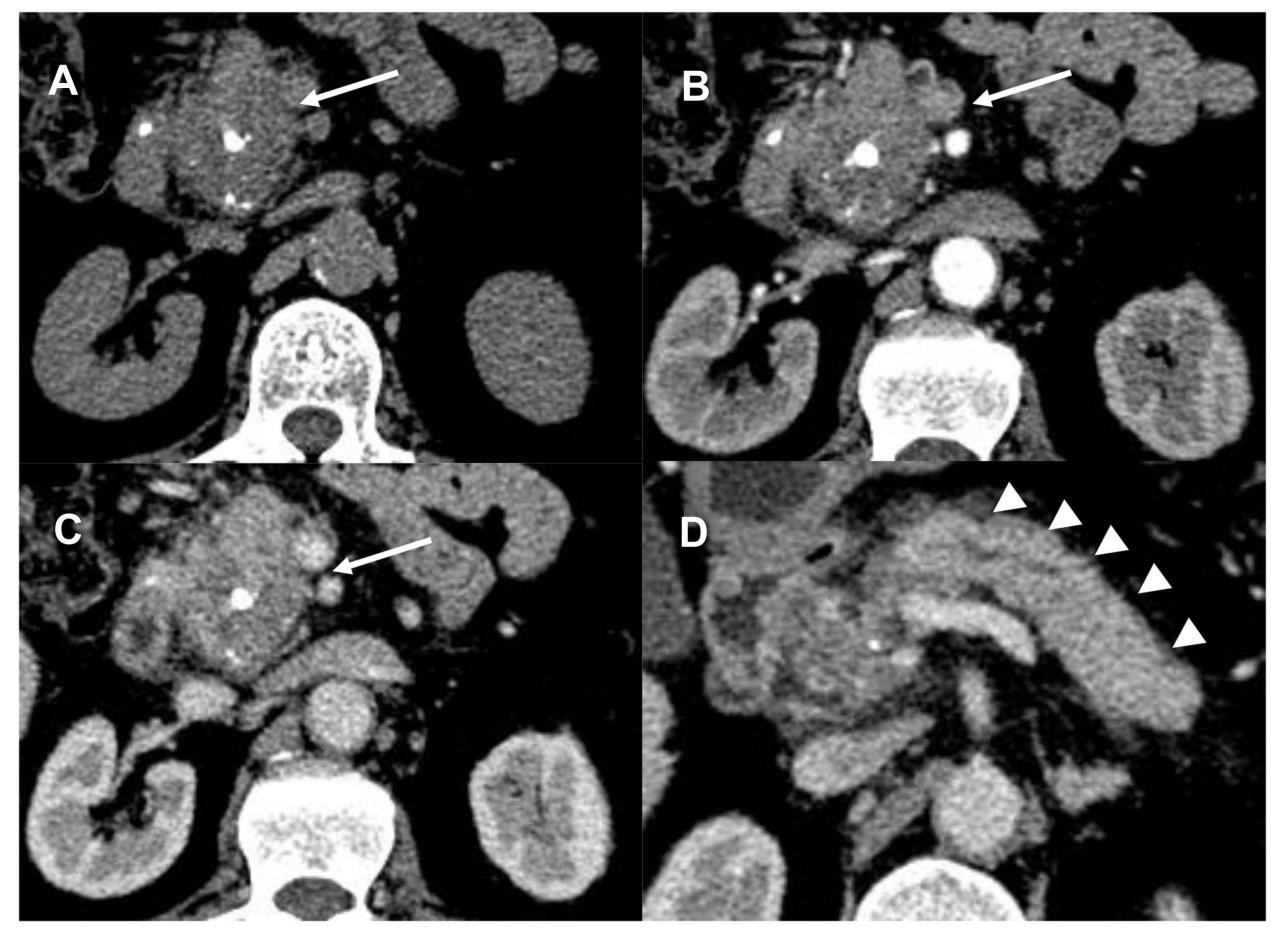

Figure 2 A 54-year-old man with mass-forming autoimmune pancreatitis. (A) Unenhanced image demonstrates an isoattenuation mass (arrow) located in the head of the pancreas and calcifications were clearly seen within the tumor. Arterial (B) and portal venous (C) phase images show an ill-defined mass (arrows) with progressive enhancement. (D) Axial CT images at the portal venous phase show diffuse enlargement of the pancreatic parenchyma (arrowheads) with slight main pancreatic duct lumen dilatation.

Abbreviation: $\mathrm{CT}$, computed tomography.

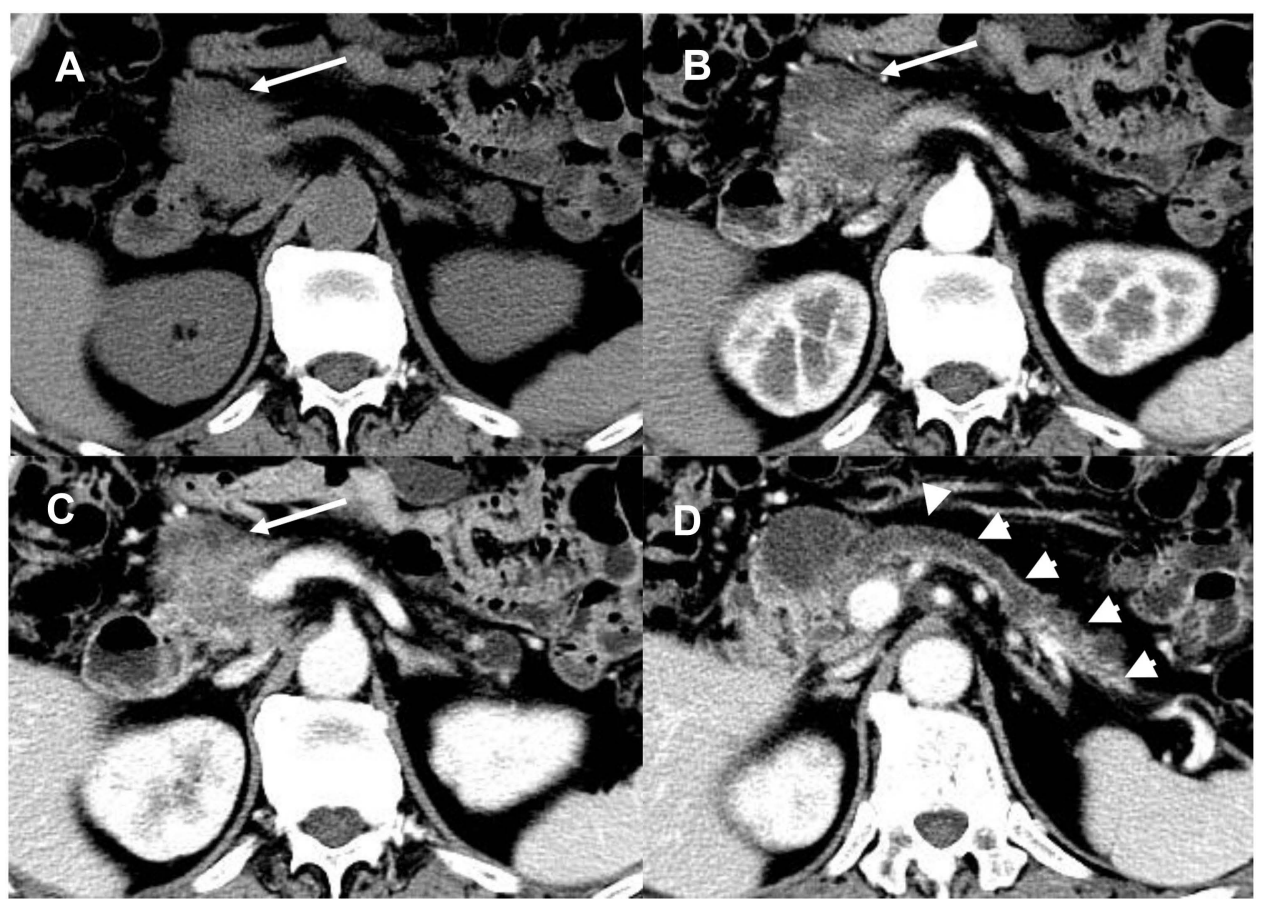

Figure 3 A 65-year-old man with pancreatic ductal adenocarcinoma. (A) Unenhanced image demonstrates an isodense mass (arrow) located in the head of the pancreas. Arterial (B) and portal venous (C) phase images show an ill-defined mass (arrows) presenting a hypovascular enhancement pattern with spleen artery invasion. (D) Main pancreatic duct lumen dilatation and pancreatic parenchymal atrophy were observed (arrowheads). 


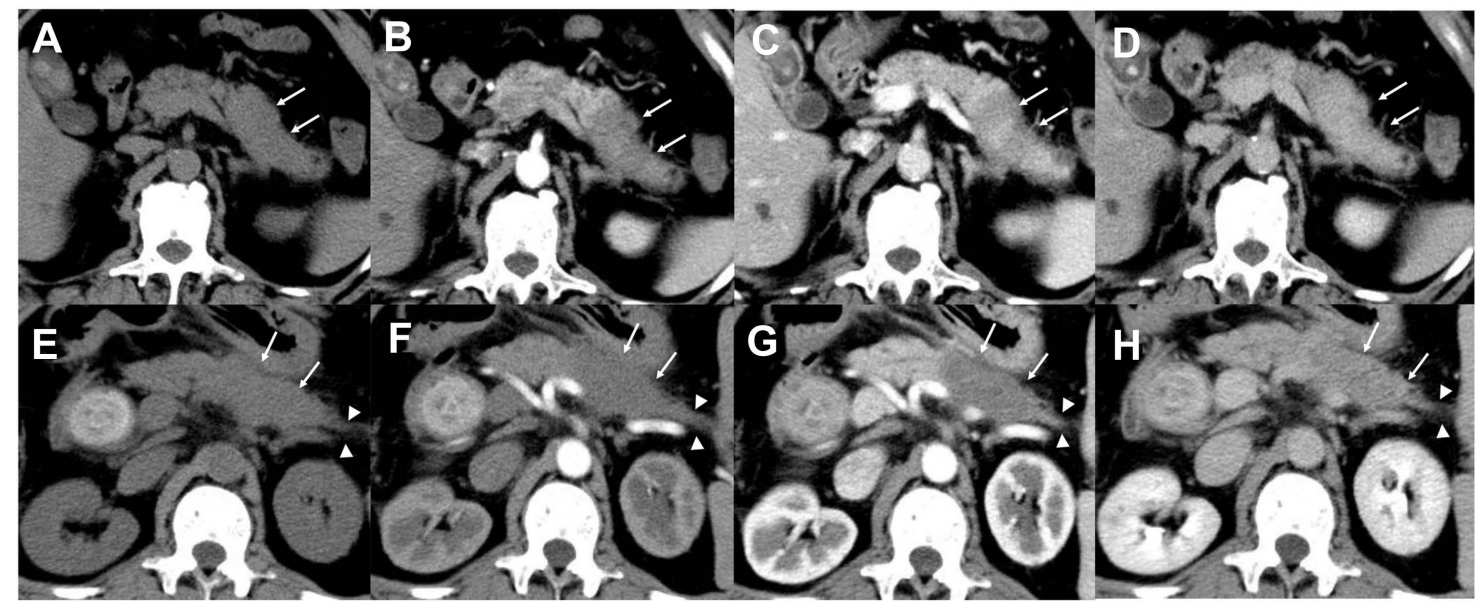

Figure 4 A 57-year-old man with chronic mass-forming pancreatitis. Unenhanced (A), arterial (B), portal venous (C), and delayed phase (D) images show a well-defined mass (arrows) in the body of the pancreas. The CT attenuation of each phase was 36, 59, 87, and $92 \mathrm{HU}$, respectively. The tumor presented a gradual enhancement pattern with no distal pancreatic parenchyma atrophy. A 68-year-old man with pancreatic ductal adenocarcinoma. Unenhanced (E), arterial (F), portal venous (G), and delayed phase (H) images show a well-defined mass (arrows) in the body of the pancreas. The CT attenuation of each phase was $35,38,46$, and $58 \mathrm{HU}$, respectively. The tumor presented a hypovascular enhancement pattern with distal pancreatic parenchyma atrophy (arrowheads).

Abbreviations: CT, computed tomography; HU, hounsfield unit.

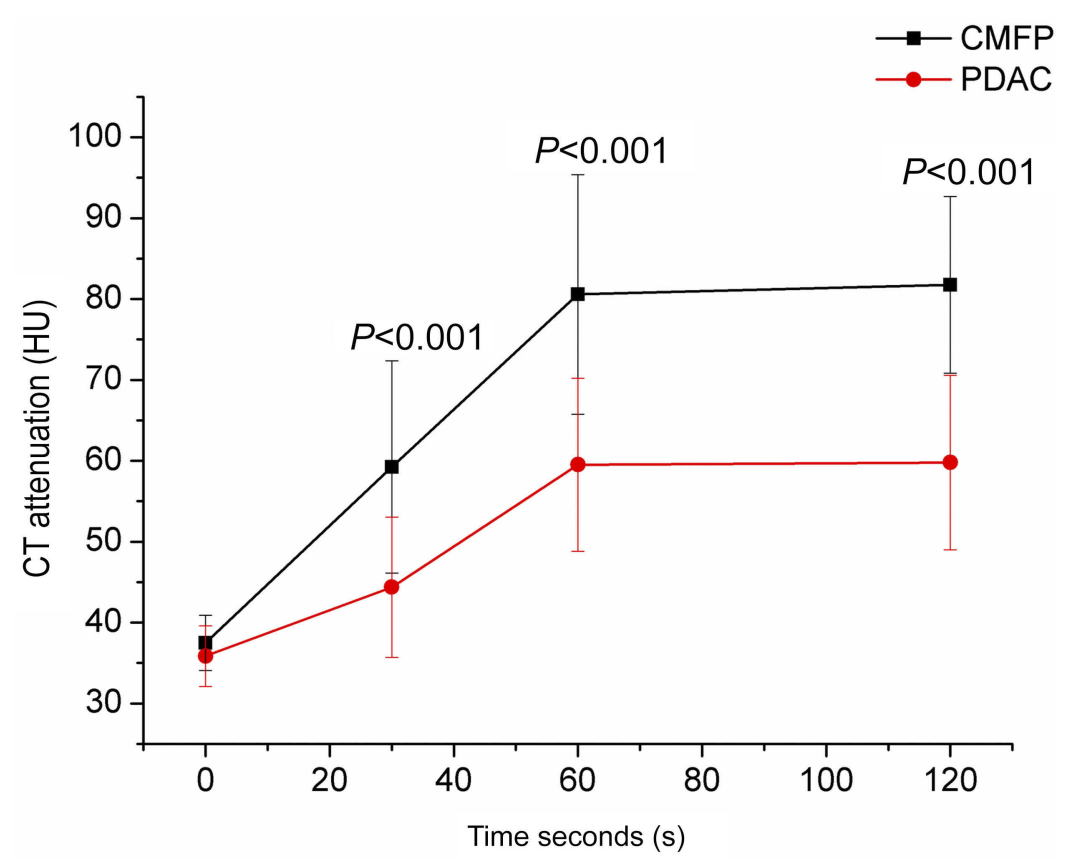

Figure 5 The dynamic contrast-enhanced curves in CMFP and PDAC. The CT attenuation of CMFP and PDAC was $37.48 \pm 4.00$ and $35.85 \pm 3.75$ Hounsfield units at the unenhanced CT images, respectively. The CT attenuation of CMFP was higher than that of PDAC at the arterial, portal venous, and delayed phase, all $P<0.05$.

Abbreviations: CMFP, chronic mass-forming pancreatitis; PDAC, pancreatic ductal adenocarcinoma; CT, computed tomography; HU, hounsfield unit.

respectively. The AUC of the combination of qualitative and quantitative variables (Model 1+2) was 0.969 (95\% CI: 0.930-1.000).

\section{Discussion}

As both PDAC and CMFP may present as a pancreatic mass, the overlap in their clinical and imaging findings makes their early preoperative and differential diagnosis difficult. ${ }^{6-9,11-13}$
Misdiagnosis of CMFP as PDAC may put extra burdens on patients and result in unnecessary intervention procedures, and misdiagnosis of PDAC as CMFP may result in delayed surgical treatment. ${ }^{20}$ Although pathological assessment of tumor identification is vital, imaging is superior in estimating the aggressiveness of the tumor and deciding a treatment plan before surgery. ${ }^{3}$ In our study, main pancreatic duct dilatation, vascular invasion, cystic necrosis, pancreatic atrophy, 


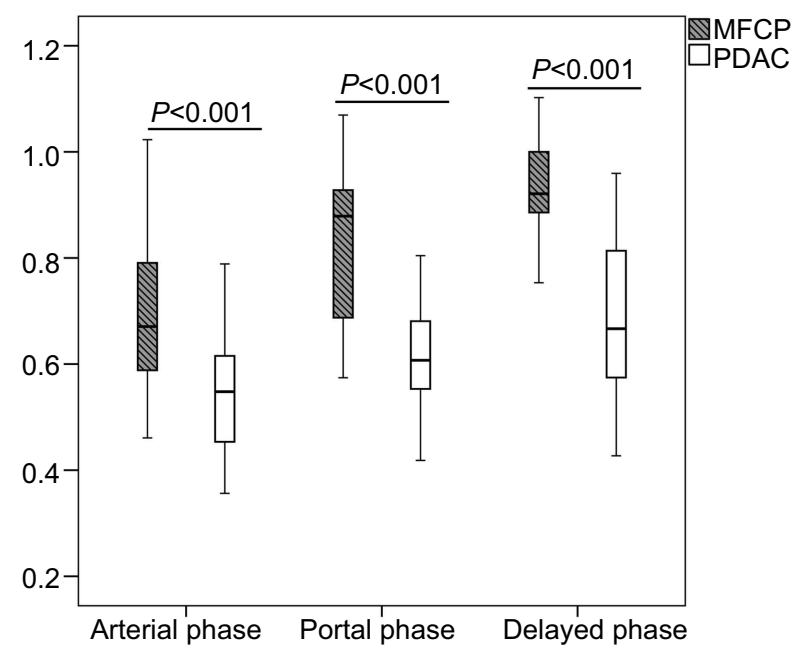

Figure 6 Box-and-whisker plots of the tumor-to-pancreas enhancement ratio in CMFP and PDAC. The tumor-to-pancreas enhancement ratios of CMFP were higher than those of PDAC at the arterial, portal venous, and delayed phase, all $P<0.05$.

Abbreviations: CMFP, chronic mass-forming pancreatitis; PDAC, pancreatic ductal adenocarcinoma.

calcification, tumor size, CT contrast enhancement, and tumor-to-pancreas enhancement ratio at the arterial phase, portal venous, and delayed phase are useful predictors for the differentiation of those two tumors.

Chronic pancreatitis is one of the risk factors in developing PDAC. In our study, 81\% of CMFP patients had a history of pancreatitis, which is much higher than that of PDAC (19.1\%). This suggests that a history of pancreatitis is an independent risk factor for CMFP, which is of great clinical significance in the diagnosis of CMFP. In addition, PDAC patients were older than CMFP patients, though statistically insignificant.

Ruan et al $^{17}$ reported that the integration of data from CE-CT, MRI, and PET/CT imaging may be helpful in distinguishing CMFP from PDAC. In our study, we evaluated the CT features of CMFP and had then compared with those of PDAC. Tummala et $\mathrm{al}^{21}$ reported that pancreatic duct dilatation is an important predictor of malignant tumors. Their study showed that 152 of 187 patients with pancreatic duct dilatation had malignant pancreatic tumors. In our study, $61.7 \%$ of PDAC patients had main pancreatic duct dilatation. However, only $33.3 \%$ of CMFP patients had main pancreatic duct dilatation, which was consistent with a previous study. Notably, the majority of duct dilatations in CMFP patients were beaded dilatation. For CMFP patients, beaded duct dilatation was secondary to chronic pancreatitis and the duct was infiltrated by inflammatory cells and fibrosis. However, PDAC originates from the ductal epithelium and duct was obstructed by infiltrative growth of the cancer resulting in distal duct dilatation. ${ }^{17}$ Choi et $\mathrm{al}^{22}$ assessed the value of contrastenhanced MRI for differentiating mass-forming autoimmune pancreatitis (AIP) from PDAC. They found that peripancreatic fat infiltration, internal cystic or necrotic portion, vascular invasion, and upstream pancreatitis were more frequent and duct penetrating sign was less frequently observed in PDAC than CMFP. In our study, we found that vascular invasion, cystic necrosis, and pancreatic atrophy were more frequent and beaded duct dilation was less frequent in PDAC compared with CMFP, which was in consistence with a previous study. In addition, we also found calcification was more common in CMFP than PDAC (33.3\% vs $10.6 \%, P=0.023)$. We speculated that it was associated with recurrent and progressive inflammatory fibrosis and accordingly resulted in calcium deposition in CMFP patients. In multivariate analysis, main duct dilatation, pancreatic atrophy, and calcification were significant predictors for differentiating CMFP from PDAC.

Ren et $\mathrm{al}^{23}$ investigated the value of CT enhancement level in differentiating duodenal gastrointestinal tumors

Table 3 Multivariate logistic regression models for prediction of chronic mass-forming pancreatitis (CMFP) vs pancreatic ductal adenocarcinoma (PDAC)

\begin{tabular}{|c|c|c|c|c|c|}
\hline Model & Features & OR (95\% Cl) & $P$-value & AUC (95\% Cl) & Cutoff value (SEN, SPE) \\
\hline Model I & $\begin{array}{l}\text { MPD } \\
\text { PA } \\
\text { Calcification }\end{array}$ & $\begin{array}{l}1.741 \text { ( } 1.085-2.792) \\
I .812(1.239-2.649) \\
0.746(0.543-1.025)\end{array}$ & $\begin{array}{l}0.03 \\
0.024 \\
0.023\end{array}$ & $0.770(0.660-0.880)$ & - \\
\hline Model 2 & $\begin{array}{l}\text { DCE } \\
\text { Size }\end{array}$ & $\begin{array}{l}0.833^{\mathrm{a}}(0.757-0.917) \\
0.15 \mathrm{I}^{\mathrm{a}}(0.028-0.8 \mathrm{I} 5)\end{array}$ & $\begin{array}{l}<0.001 \\
0.028\end{array}$ & $0.943(0.888-0.999)$ & $\begin{array}{l}70.5(84.2,84.7) \\
3.285(1,59.6)\end{array}$ \\
\hline \multicolumn{2}{|c|}{ Model I + Model 2} & 1.991 (I.460-2.714) & $<0.001$ & $0.969(0.930-1.000)$ & $-(84.2,94.7)$ \\
\hline
\end{tabular}

Note: ${ }^{a}$ Data are adjusted ORs per one SD change.

Abbreviations: MPD, main pancreatic duct dilatation; PA, pancreatic atrophy; DCE, delayed contrast enhancement; SEN, sensitivity; SPE, specificity. 


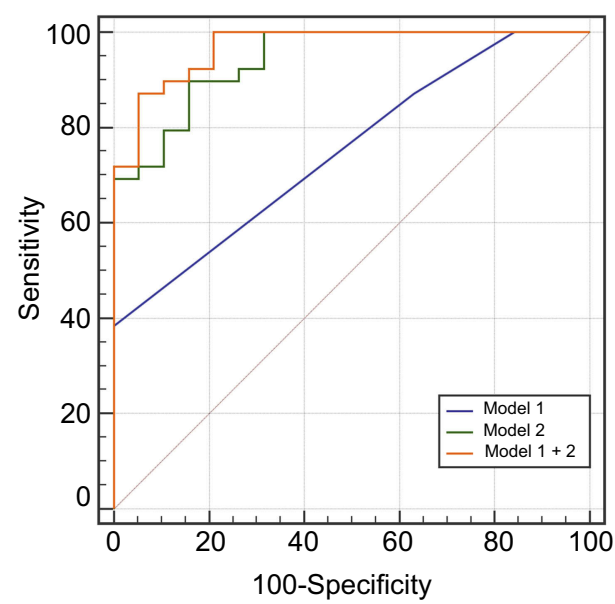

Figure 7 Receiver operating characteristic curve for diagnostic performance of CT regarding the differentiation between CMFP (chronic mass-forming pancreatitis) and PDAC (pancreatic ductal adenocarcinoma) with qualitative (Model I), quantitative (Model 2), and combination of qualitative and quantitative CT imaging features (Model I+2). The areas under the curve of which are $0.770,0.943$, and 0.969

from hypervascular pancreatic neuroendocrine tumors. Takumi et $\mathrm{al}^{24}$ assessed the value of CT contrast enhancement and tumor-to-pancreas ratio in pancreatic neuroendocrine tumors grading. In our study, we evaluated the diagnostic performance of tumor size, contrast enhancement, and tumor-to-pancreas enhancement ratio at the arterial, portal venous, and delayed phase in differentiating CMFP from PDAC. Tumor size and delayed contrast enhancement were significant predictors for differentiating CMFP from PDAC, the cutoff values of which were 3.285 cm with $100 \%$ sensitivity and $50.9 \%$ specificity and 70.5 HU with $84.2 \%$ sensitivity and $84.7 \%$ specificity, respectively.

There were several possible limitations in our study. First, CT scanners used in our study were various due to its retrospective nature. However, contrast media and CT scanning parameters used in our study were similar. Second, some inconformity during image analysis was resolved by referral to a third radiologist. Third, we used a fixed-delay method for CE-CT, and arterial phase scanning began $30 \mathrm{~s}$ after contrast media injection, not the pancreatic parenchymal phase (35-40 s). However, the purpose of our study is to provide information for prompt decision-making. Fourth, only CE-CT was investigated. The role of other imaging modalities, including endoscopic ultrasound-guided fine-needle aspiration (EUSFNA), diffusion-weighted imaging (DWI), and PET-CT, was not included in our study. Further studies addressing the diagnostic reliability of other imaging modalities or comparisons between $\mathrm{CT}$ and other imaging modalities are needed. Fifth, the number of enrolled patients is too small. Chronic pancreatitis was common in clinical practice, but only a few chronic pancreatitis presented as CMFP, ${ }^{25}$ and we could not collect more CMFP in a short-term period to increase our patient enrollment due to its low incidence. In the future, we are planning to conduct a multicenter program to collect more CMFP patients to increase the sample size and make an external validation for the confirmation of the diagnostic value of the qualitative and quantitative CT predicting models in discriminating CMFP from PDAC.

\section{Conclusion}

In conclusion, main pancreatic duct dilatation, vascular invasion, cystic necrosis, pancreatic atrophy, and calcification were shown to be useful CT imaging features in discriminating CMFP from PDAC. Moreover, our data indicated that quantitative CT parameters, especially tumor size and tumor contrast enhancement at the delayed phase, played an important role in differentiating those two tumors. Tumor size $>3.285 \mathrm{~cm}$ and delayed contrast enhancement $>70.5 \mathrm{HU}$ are indicative of CMFP. The combination of qualitative and quantitative variables in differentiating CMFP and PDAC showed excellent performance. This information could be used to support decisions considering the extent of tumor resection or the possibility of a conservative approach.

\section{IRB statement}

This study was approved by the institutional review board of the Affiliated Hospital of Nanjing University of Chinese Medicine and patient informed consent was waived due to its retrospective nature. All analysis of confidential data was carried out in accordance with the Declaration of Helsinki principles.

\section{Acknowledgments}

We thank all authors for their continuous and excellent support with patient data collection, imaging analysis, statistical analysis and valuable suggestions for the article. This study was supported by the Key Program of Research and Development of Jiangsu Province (BE2017772) and the National Natural Science Foundation of China (81771899).

\section{Disclosure}

The authors report no conflicts of interest in this work. 


\section{References}

1. Kleeff J, Korc M, Apte M, et al. Pancreatic cancer. Nat Rev Dis Primers. 2016;2:16022. doi:10.1038/nrdp.2016.22

2. Conroy T, Van Laethem JL. Combination or single-agent chemotherapy as adjuvant treatment for pancreatic cancer? Lancet Oncol. 2019;20(3):336-337. doi:10.1016/S1470-2045(19)30310-9

3. Ren S, Chen X, Wang Z, et al. Differentiation of hypovascular pancreatic neuroendocrine tumors from pancreatic ductal adenocarcinoma using contrast-enhanced computed tomography. PLoS One. 2019;14(2):e0211566. doi:10.1371/journal.pone.0211566

4. Gu X, Liu R. Application of 18f-FDG PET/CT combined with carbohydrate antigen 19-9 for differentiating pancreatic carcinoma from chronic mass-forming pancreatitis in Chinese elderly. Clin Interv Aging. 2016;11:1365-1370. doi:10.2147/CIA.S115254

5. Weiss VL, Kiernan C, Wright J, et al. Fine-needle aspiration-based grading of pancreatic neuroendocrine neoplasms using $\mathrm{Ki}-67$ : is accurate WHO grading possible on cytologic material? $\mathrm{J} \mathrm{Am} \mathrm{Soc}$ Cytopathol. 2018;7(3):154-159. doi:10.1016/j.jasc.2018.02.001

6. Shi Y, Gao F, Li Y, et al. Differentiation of benign and malignant solid pancreatic masses using magnetic resonance elastography with spin-echo echo planar imaging and three-dimensional inversion reconstruction: a prospective study. Eur Radiol. 2018;28(3):936945. doi:10.1007/s00330-017-5062-y

7. Shi Y, Cang L, Zhang X, et al. The use of magnetic resonance elastography in differentiating autoimmune pancreatitis from pancreatic ductal adenocarcinoma: a preliminary study. Eur $J$ Radiol. 2018;108:13-20. doi:10.1016/j.ejrad.2018.09.001

8. Harmsen FR, Domagk D, Dietrich CF, Hocke M. Discriminating chronic pancreatitis from pancreatic cancer: contrast-enhanced EUS and multidetector computed tomography in direct comparison. Endosc Ultrasound. 2018;7(6):395-403. doi:10.4103/eus.eus_24_18

9. Zhang TT, Wang L, Liu H, et al. Differentiation of pancreatic carcinoma and mass-forming focal pancreatitis: qualitative and quantitative assessment by dynamic contrast-enhanced MRI combined with diffusion-weighted imaging. Oncotarget. 2017;8(1):1744-1759. doi:10.18632/oncotarget. 12120

10. Muhi A, Ichikawa T, Motosugi U, et al. Mass-forming autoimmune pancreatitis and pancreatic carcinoma: Differential diagnosis on the basis of computed tomography and magnetic resonance cholangiopancreatography, and diffusion-weighted imaging findings. J Magn Reson Imaging. 2012;35(4):827-836. doi:10.1002/jmri.22881

11. Prignon A, Provost C, Alshoukr F, et al. Preclinical evaluation of GaDOTA-NT-20.3: a promising PET imaging probe to discriminate human pancreatic ductal adenocarcinoma from pancreatitis. Mol Pharm. 2019;16 (6):2776-2784. doi:10.1021/acs.molpharmaceut.9b00283

12. Frøkjær JB, Akisik F, Farooq A, et al. Guidelines for the diagnostic cross sectional imaging and severity scoring of chronic pancreatitis. Pancreatology. 2018;18(7):764-773. doi:10.1016/j.pan.2018.06.002
13. Yadav AK, Sharma R, Kandasamy D, et al. Perfusion CT-Can it resolve the pancreatic carcinoma versus mass forming chronic pancreatitis conundrum? Pancreatology. 2016;16(6):979-987. doi:10.1016/j.pan.2016.08.011

14. Aslan S, Nural MS, Camlidag I, Danaci M. Efficacy of perfusion CT in differentiating of pancreatic ductal adenocarcinoma from massforming chronic pancreatitis and characterization of isoattenuating pancreatic lesions. Abdom Radiol. 2019;44(2):593-603. doi:10. 1007/s00261-018-1776-9

15. Yin Q, Zou X, Zai X, et al. Pancreatic ductal adenocarcinoma and chronic mass-forming pancreatitis: differentiation with dual-energy MDCT in spectral imaging mode. Eur J Radiol. 2015;84(12):24702476. doi:10.1016/j.ejrad.2015.09.023

16. Kim DW, Kim HJ, Kim KW, et al. Neuroendocrine neoplasms of the pancreas at dynamic enhanced CT: comparison between grade 3 neuroendocrine carcinoma and grade $1 / 2$ neuroendocrine tumour. Eur Radiol. 2015;25(5):1375-1383. doi:10.1007/s00330-014-3532-z

17. Ruan Z, Jiao J, Min D, et al. Multi-modality imaging features distinguish pancreatic carcinoma from mass-forming chronic pancreatitis of the pancreatic head. Oncol Lett. 2018;15(6):9735-9744.

18. Guo C, Zhuge X, Wang Z, et al. Textural analysis on contrastenhanced CT in pancreatic neuroendocrine neoplasms: association with WHO grade. Abdom Radiol. 2019;44(2):576-585. doi:10.1007/ s00261-018-1763-1

19. Ahn SS, Kim MJ, Choi JY, et al. Indicative findings of pancreatic cancer in prediagnostic CT. Eur Radiol. 2009;19(10):2448-2455. doi:10.1007/s00330-009-1422-6

20. Dutta AK, Chacko A. Head mass in chronic pancreatitis: Inflammatory or malignant. World J Gastrointest Endosc. 2015;7 (3):258-264. doi:10.4253/wjge.v7.i3.258

21. Tummala P, Rao S, Agarwal B. Differential diagnosis of focal noncystic pancreatic lesions with and without proximal dilation of pancreatic duct noted on CT scan. Clin Transl Gastroenterol. 2013;4:e42. doi:10.1038/ctg.2013.15

22. Choi SY, Kim SH, Kang TW, et al. Differentiating mass-forming autoimmune pancreatitis from pancreatic ductal adenocarcinoma on the basis of contrast-enhanced MRI and DWI findings. AJR Am J Roentgenol. 2016;206(2):291-300. doi:10.2214/AJR.15.14974

23. Ren S, Chen X, Wang J, et al. Differentiation of duodenal gastrointestinal stromal tumors from hypervascular pancreatic neuroendocrine tumors in the pancreatic head using contrast-enhanced computed tomography. Abdom Radiol. 2019;44(3):867-876. doi:10.1007/s00261-018-1803-x

24. Takumi K, Fukukura Y, Higashi M, et al. Pancreatic neuroendocrine tumors: correlation between the contrast-enhanced computed tomography features and the pathological tumor grade. Eur $J$ Radiol. 2015;84(8):1436-1443. doi:10.1016/j.ejrad.2015.05.005

25. Jee KN. Mass forming chronic pancreatitis mimicking pancreatic cystic neoplasm: a case report. World J Gastroenterol. 2018;24 (2):297-302. doi:10.3748/wjg.v24.i2.297

\section{Publish your work in this journal}

Cancer Management and Research is an international, peer-reviewed open access journal focusing on cancer research and the optimal use of preventative and integrated treatment interventions to achieve improved outcomes, enhanced survival and quality of life for the cancer patient.
The manuscript management system is completely online and includes a very quick and fair peer-review system, which is all easy to use. Visit http://www.dovepress.com/testimonials.php to read real quotes from published authors. 